\title{
Effects of Refuge Prey on Stability of the Prey-Predator Model Subject to Immigrants: A Mathematical Modelling Approach
}

\author{
Mussa Amos Stephano ${ }^{1}$ and II Hyo Jung ${ }^{2}$ \\ ${ }^{1}$ Mkwawa University College of Education, P. O. Box 2513, Iringa Tanzania \\ ${ }^{2}$ Pusan National University, P. O. Box 46241, Busan, Republic of Korea \\ E-mail: mussaamos314@gmail.com \\ Received 21 Jun 2021, Revised 20 Sep 2021, Accepted 25 Sep 2021, Published Oct 2021 \\ DOI: https://dx.doi.org/10.4314/tjs.v47i4.4
}

\begin{abstract}
Prey-predator system is enormously complex and nonlinear interaction between species. Such complexity regularly requires development of new approaches which involves more factors in analysis of its population dynamics. In this paper, we formulate a modified Lotka-Volterra model that incorporates factors such as refuge prey and immigrants. We investigate the effects of refuge prey and immigrants by varying the refuge factor, with and without immigrants. The results show that with Holling's type I functional response, the proposed model is asymptotically convergent when a refuge prey factor is introduced. Moreover, with Holling's type II functional response, the proposed mathematical model is unstable and does not converge. However, with Holling's type III functional response in a system, the proposed mathematical model is asymptotically stable. These results point out the following remarks: The effects of refuge prey on stability of the dynamical system vary depending on the type of functional response, and when the predator population increases, the likelihood of prey extinction declines when the proportion of preys in refuge population increases. Hence, the factor of refuge prey is crucial for controlling the population of the predator and obtaining balances between prey and predator in the ecosystem.
\end{abstract}

Keywords: Refuge prey, stability, prey-predator, immigrants, Mathematical modelling.

\section{Introduction}

Ecology is the scientific study that deals with interactions, dynamical relationship, abundance, distribution of organisms and biodiversity among them in their biophysical habitats (Cohen 1977, Brown and Roughgarden 1990). It is a wide term that constitutes of many fields, including ecosystem, aquatic, terrestrial, microbial, population and physiology (Wertheim et al. 2005). In both ecology and environmental science, a fundamental theme is the energetic interconnections among them. Living organisms are interrelated, whereby living is naturally dependent based on different factors. There are several factors that have significant impacts on stability of population, including predation, immigration and refuge.

Predation is defined as biological interaction between two or more organisms such as prey and predator. There are various types of predation based on classification of nature. A predator is living organism that ingests another organism, while a prey is the living organism which a predator ingests. Examples of prey-predators are zebra-lion, gazelles-lion, fish-shark, leaf-grasshopper, fishbear, rabbit-fox, and insects-birds. The aforementioned relationships are ecologically 
potential and prime movers of energy from one organism to another (Petchey 2000, Allen 2007). Moreover, the prey-predator relationships continue to be beneficial in forcing prey species to adapt and ensure that they feed without becoming a meal for another predator (May 1993, Hansson and Åkesson 2014).

Immigration refers to an organism establishes particular habitat. Both immigration and migration of animals occur when there is scarcity of basic needs sometimes due to human activities in wildlife habitats (Singh and Singh 1999, Holtmeier 2015). Speccifically, preypredator interactions have been distrupted due to different responses on the climate change (Parmesan 2006). Regular and seasonal migration and immigration drive living organisms to adopt travelling and movement lives (Thomas 2019).

Refuge refers to an inaccessibility of prey by predator in their areas as means of protection (Sih 1987). Both prey and predator population densities are significantly high when refuges are available. The idea of refuge has a significant impact in ecology and population dynamics of prey and predator as a stabilizing force (Berryman 2006). Moreover, refuges in population dynamics have both stabilizing and destabilizing impacts in prey-predator interactions (Robinson et al. 2013).

Mathematical modelling is an integral and scientific tool that is used to investigate factors that have significant impacts on stability of dynamical system. Prey-predator's mathematical models have been formulated and analysed, these include the work of Cohen (1977), who provided a scientific research of population oscillations. Subsequently, they were followed by the theoretical work of Lotka-Volterra for population fluctuations and time-series analysis. Alinhac (1995) and Mukherjee (2016) described an Ordinary Differential Equation (ODE) and Lotka-Volterra model with immigration factor is presented. T he effects influenced by immigrants on stability to a prey-predator population were analysed by Kar (2005) and Takeru et al. (2018). However, no study has instantly used all three factors, namely predation, immigration and refuge. In this paper, we present an integration of Mukherjee (2016), Takeru et al. (2018) and Kar (2005) by formulating a deterministic mathematical model and analyse the effects of refuge prey. This paper organized as follows: In the next section, the mathematical model of preypredator is derived, followed by sections devoted to model analysis and numerical simulation, and finally conclusion.

\section{Materials and Methods \\ Model formulation}

The integration of models by Mukherjee (2016), Takeru et al. (2018) and Kar (2005), the preypredator model consists of two populations $x$ and $y$, namely prey and predator, respectively. The relationship between density of consumed prey per predator and numerical responses in their densities is referred to a functional response of prey-predator. The functional responses are generally classified as prey density-dependent $f(x)$ ratio-dependent $f(x / y)$ and prey-predator density dependent $f(x, y$. Denny (2014) carried out an experimental work on interactions and predator's rates of prey capture. The outcomes are famously known as Holling's type I, II and III functional responses. The interaction of species $\mathrm{x}$ and $\mathrm{y}$ is referred to functional response based on the nature and traits of population density;

$$
f(x, y)=\frac{a x^{1+\alpha} y}{1+h x^{1+\alpha}}
$$

Where $h$ and $\alpha$ are functional response coefficients which are handling time and Hill exponent, respectively. If $h=0, \alpha=0$, then

$$
f(x, y)=a x y
$$

is called Holling's type I functional response which is linear in nature and practically 
acceptable and found in passive predators like spiders. If $h \neq 0, \alpha=0$, then

$$
\mathrm{f}(\mathrm{x}, \mathrm{y})=\frac{\mathrm{axy}}{1+\mathrm{hx}}
$$

is called Holling's type II functional response with hyperbolic property and respond positively in vertebrates. If $h \neq 0, \alpha=1$, then

$$
f(x, y)=\frac{a x^{2} y}{1+h x^{2}}
$$

is Holling's type III functional response or sigmoid functional response.

In this study, we consider a consistent habitat of prey and predator population and formulate a mathematical model based on the following assumptions. The population density of prey represented by $x(t)$ at any time $t \geq 0$ and population density of predator represented by $y(t)$. In absence of predators, preys increase exponentially without interacting with predators. $x(t), y(t), t \geq 0$, where $y(t)=$ 0 , predators will disappear by starving, if there are no preys in their habitats, as $t \rightarrow \infty, y(t) \rightarrow$ 0 if and only if $x(t)=0$. The rate of growth of prey is proportional to their population size, and the rate growth of predators depends on the increase of the prey population size. The interaction of prey and predator is shown in Figure 1, and the model parameters are described in Table 1.

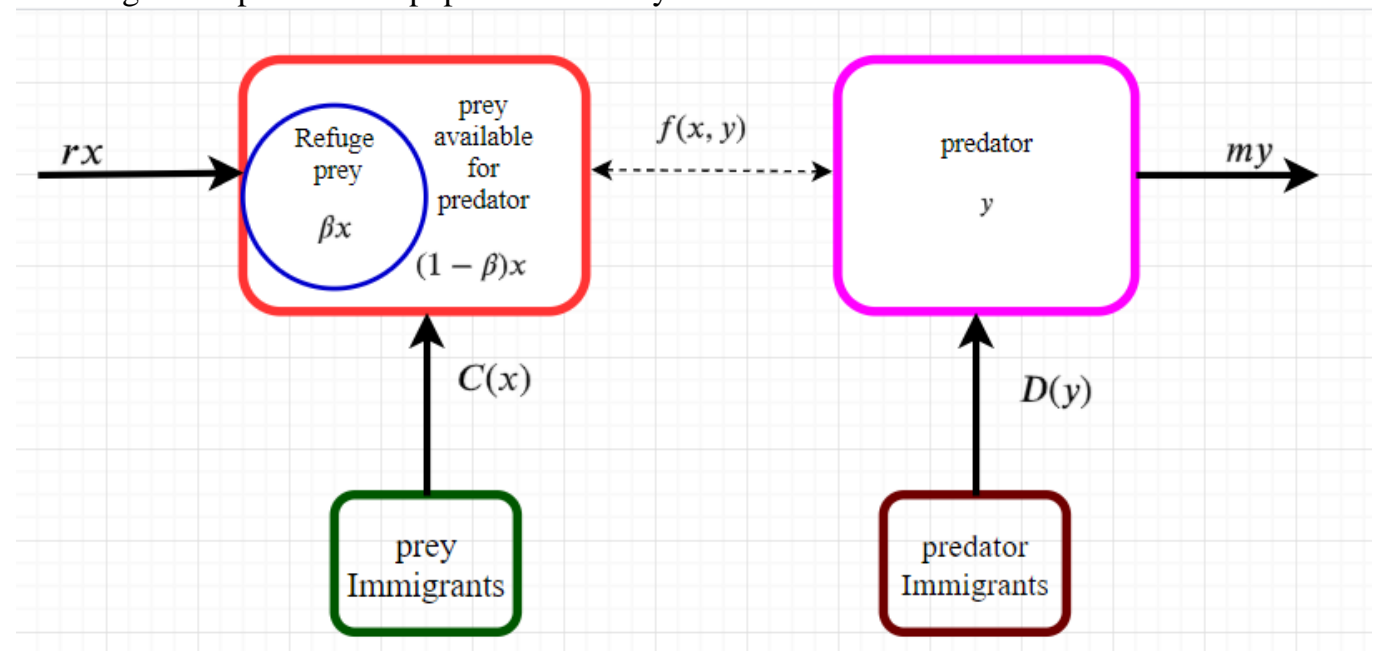

Figure 1: Schematic diagram of prey and predator interaction.

Table 1: Description of model parameters

\begin{tabular}{cl}
\hline Parameter & Description \\
\hline$r$ & Intrinsic growth rate of prey \\
$a$ & Rate at which predator catches prey \\
$\beta$ & Rate of refuge prey \\
$b$ & Conversion factor denoting the number of newly born predators for \\
& each captured prey \\
$m$ & Death rate of predator \\
$d$ & Small positive immigration factor into prey population \\
$h$ & Small positive immigration factor into predator population \\
\hline
\end{tabular}


The dynamics of prey-predator interactions is described by the following system of ordinary differential equations:

$$
\begin{aligned}
& \left\{\begin{array}{l}
\frac{d x}{d t}=r x-\frac{a[(1-\beta) x]^{1+\alpha} y}{1+h[(1-\beta) x]^{1+\alpha}}+C(x) \\
\frac{d y}{d t}=\frac{b[(1-\beta) x]^{1+\alpha} y}{1+h[(1-\beta) x]^{1+\alpha}}-m y+D(y)
\end{array}\right. \\
& \text { where } C(x)=\left\{\begin{array}{l}
c \geq 0 \\
\frac{c}{x}>0
\end{array} \text { and } D(x)=\left\{\begin{array}{l}
d \geq 0 \\
\frac{d}{x}>0
\end{array}\right.\right.
\end{aligned}
$$

With initial conditions: $x(0)>0$ and $y(0)>$ 0 .

\section{Model analysis}

The model system (5) is mathematically and biologically meaningful if solutions are nonnegative. In this section, we investigate positivity of model solutions.

\section{Positivity of model solution}

$$
\frac{d x}{d t}=r x-\frac{a[(1-\beta) x]^{1+\alpha} y}{1+h[(1-\beta) x]^{1+\alpha}}+C(x)
$$

Separation of variables leads to;

$$
\frac{d x}{d t} \geq-\frac{a[(1-\beta) x]^{1+\alpha} y}{1+h[(1-\beta) x]^{1+\alpha}}
$$

Integration and application of initial condition yields

$$
\begin{aligned}
& x(t) \\
& \geq x(0) \exp \int_{0}^{t} \frac{a(1-\beta)^{1+\alpha} x(s)^{\alpha} y(s)}{1+h[(1-\alpha) x(s)]^{1+\alpha}} d s
\end{aligned}
$$$$
\geq 0 \text {. }
$$

Applying the same approach for rest of the equation, we obtain

$$
y(t) \geq y(0) e^{-m t} \geq 0
$$

\section{Coexistence equilibrium points}

We discussed the existence of equilibrium points specifically on positive or interior equilibrium. However, a trivial equilibrium point $E_{0}(0,0)$ represents extinction equilibrium. Interior equilibrium points $E_{i}\left(x^{*}, y^{*}\right)$ where $i=1,2, \ldots, 12$. are evaluated by setting $\frac{d x}{d t}=0$ and $\frac{d y}{d t}=0$ as follows:

Case 1: model system (5) with Holling's type I functional response when $C(x)=c$ and $D(y)=0$. The model system (5) is reduced to;

$$
\begin{gathered}
\frac{d x}{d t}=r x^{*}-a(1-\beta) x^{*} y^{*}+c=0 \\
\frac{d y}{d t}=b(1-\beta) x^{*} y^{*}-m y^{*}=0
\end{gathered}
$$

By using second equation to evaluate $x^{*}$

$$
\begin{gathered}
\Rightarrow b(1-\beta) x^{*} y^{*}-m y^{*}=0 \\
\Rightarrow x^{*}=\frac{m}{b(1-\beta)}
\end{gathered}
$$

Direct substitution of $x^{*}$ into first equation gives $y^{*}=\left(\frac{m r+b c}{a m(1-\beta)}\right)$

$$
x^{*}, y^{*}=\frac{m}{b(1-\beta)}, \quad \frac{m r+b c}{a m(1-\beta)}
$$

Applying the same approach for the rest of the equation, we obtain the detailed table (Table 2) for all twelve cases.

The general Jacobian matrix from the model system (5) with Holling's type I, II and III functional responses is given by;

$$
J(x, y)=\left(\begin{array}{lr}
r-\frac{a(1+\alpha)(1-\beta)^{1+\alpha} x^{\alpha} y}{\left(1+h[(1-\beta) x]^{1+\alpha}\right)^{2}}+\frac{\partial C(x)}{\partial x} & -\frac{a[(1-\beta) x]^{1+\alpha}}{1+h[(1-\beta) x]^{1+\alpha}} \\
\frac{b(1+\alpha)(1-\beta)^{1+\alpha} x^{\alpha} y}{\left(1+h[(1-\beta) x]^{1+\alpha}\right)^{2}} & \frac{a[(1-\beta) x]^{1+\alpha}}{1+h[(1-\beta) x]^{1+\alpha}}-m+\frac{\partial D(y)}{\partial y}
\end{array}\right)
$$

Specifically, at equilibrium point $E_{1}$, the condition to be positive $0 \leq \beta<1+\frac{m r}{b c}$ must hold. Consequently, $\lambda^{2}+\frac{b c(1-\beta)}{m} \lambda+m r+$ $b c(1-\beta)=0$ is a characteristic equation, applying the same approach for rest of equilibrium points. 
Stephano and Jung - Effects of refuge prey on stability of the prey-predator model ...

Table 2: Equilibria of the mathematical model (5) in twelve cases of coexistence

\begin{tabular}{|c|c|c|}
\hline Model & Immigration factor & Equilibrium points \\
\hline $\begin{array}{l}\frac{d x}{d t}=r x-a(1-\beta) x y+C(x) \\
\frac{d y}{d t}=b(1-\beta) x y-m y+D(y)\end{array}$ & $\begin{array}{l}C(x)=c, D(y)=0 \\
C(x)=0, D(y)=d \\
C(x)=\frac{c}{x}, D(y)=0 \\
C(x)=0, D(y)=\frac{d}{y}\end{array}$ & $\begin{array}{l}x^{*}=\frac{m}{b(1-\beta)}, y^{*}=\frac{m u+b c \cdot(1-\rho)}{m a(1-\beta)} \\
x^{*}=\frac{m r-d a(1-\beta)}{b r(1-\beta)}, y^{*}=\frac{r}{a(1-\beta)} \\
x^{*}=\frac{m}{b(1-\beta)}, y^{*}=\frac{m r^{2}+c b^{2}(1-\beta)^{2}}{m^{2} a(1-\beta)} \\
x^{*}=\frac{m r^{2}-d a^{2}(1-\beta)^{2}}{r^{2} b(1-\beta)}, y^{*}=\frac{r}{a(1-\beta)}\end{array}$ \\
\hline $\begin{array}{l}\frac{d x}{d t}=r x-\frac{a(1-\beta) x y}{1+h(1-\beta) x}+C(x) \\
\frac{d y}{d t}=\frac{b(1-\beta) x y}{1+h(1-\beta) x}-m y+D(y)\end{array}$ & $\begin{array}{l}C(x)=c, D(y)=0 \\
C(x)=0, D(y)=d \\
C(x)=\frac{c}{x}, D(y)=0 \\
C(x)=0, D(y)=\frac{d}{y}\end{array}$ & $\begin{array}{l}x^{*}=\frac{m}{(1-\beta)(b-h m)}, \\
y^{*}=\frac{b(m r+c(1-\beta)(b-h m))}{m a(1-\beta)(b-h m)} \\
x^{*}=\frac{d a(1-\beta)-m r}{(1-\beta)(m h-b) r}, \\
y^{*}=\frac{r\left(1+h(1-\beta) x^{*}\right)}{a(1-\beta)} \\
x^{*}=\frac{m}{(1-\beta)(b-h m)}, \\
y^{*}=\frac{\left(r x^{* 2}+c\right)\left(1+x^{*}(h(1-\beta))\right)}{x^{* 2}} \\
x^{*}=\frac{-B \pm \sqrt{B^{2}-4 A C}}{2 A} \\
y^{*}=\frac{r\left(1+h(1-\beta) x^{*}\right)}{a(1-\beta)} \\
A=r^{2} h(1-\beta)^{2}(b-h m) \\
B=r^{2} h(1-\beta)\left(1-m^{2}\right) \\
C=d a^{2}(1-\beta)^{2}-r^{2} m\end{array}$ \\
\hline $\begin{array}{l}\frac{d x}{d t}=r x-\frac{a(1-\beta)^{2} x^{2} y}{1+h(1-\beta)^{2} x^{2}}+C(x) \\
\frac{d y}{d t}=\frac{b(1-\beta)^{2} x^{2} y}{1+h(1-\beta)^{2} x^{2}}-m y+D(y)\end{array}$ & $\begin{array}{l}C(x)=c, D(y)=0 \\
C(x)=0, D(y)=d \\
C(x)=\frac{c}{x}, D(y)=0 \\
C(x)=0, D(y)=\frac{d}{y}\end{array}$ & $\begin{array}{l}x^{*}= \pm \sqrt{\frac{m}{(1-\beta)^{2}(b-h m)},} \\
y^{*}=\frac{\left(r+\frac{c}{\left.x^{*}\right)\left(1+h(1-\beta)^{2} x^{* 2}\right)}\right.}{a(1-\beta)^{2} x^{*}} \\
x^{*}=\frac{a \pm \sqrt{a^{2}+\frac{4(b r-h m r) m r}{1-\beta}}}{2(h m r-b r)} \\
y^{*}=\frac{r\left(1+h(1-\beta)^{2} x^{* 2}\right)}{a(1-\beta)^{2}} x^{*} \\
x^{*}= \pm \sqrt{\frac{m}{(1-\beta)^{2}(b-h m)}}, \\
y^{*}=\frac{\left(r+\frac{c}{x^{* 2}}\right)\left(1+h(1-\beta)^{2} x^{* 2}\right)}{a(1-\beta)^{2} x^{*}} \\
x^{*}= \pm \sqrt{\frac{-B_{1} \pm \sqrt{B_{1}^{2}-4 A_{1} C_{1}}}{2 A_{1}}}, \\
y^{*}=\frac{r\left(1+h(1-\beta)^{2} x^{* 2}\right)}{a(1-\beta)^{2} x^{*}} \\
A_{1}=-m r^{2} h^{2}(1-\beta)^{3}, \\
B_{1}=b(1-\beta)^{2}\left(b+(1-\beta)^{2} d a^{2}-\right. \\
\left.2 m r^{2} h\right) \\
C_{1}=-m r^{2}\end{array}$ \\
\hline
\end{tabular}

\section{Numerical simulation}

To understand the dynamics of the prey and predator, the deterministic mathematical model (5) is simulated in MATLAB using the following parameters: $\beta=[0,0.01,0.1,0.4,0.7,0.9]$ in range of $0 \leq \beta<1, \quad r=a=0.1, b=0.3$, $m=0.2$, and $h=0.1$ with initial conditions of $x_{0}=5$ and $y_{0}=5$ are used.

Figures 2-5 represent the simulated results of our proposed model with
Holling's type I functional response with and without refuge prey factor. Precisely, when $0<\beta \leq r$, there is a neutral effect on the stability which implies the protected prey will exist, and when $r<\beta<1$, the system is disturbed. Consequently, requires more time to converge asymptotically. Figures 6-9 present the system with Holling's type II functional response, with increases of $\beta$ leading to a growing periodic 
behaviour which destabilizes the system by no convergence. Figure 10 represents Holling's type III functional response has promising results, showing that the system is asymptotically convergent in a range $0 \leq \beta<1$ making it stable. (a)
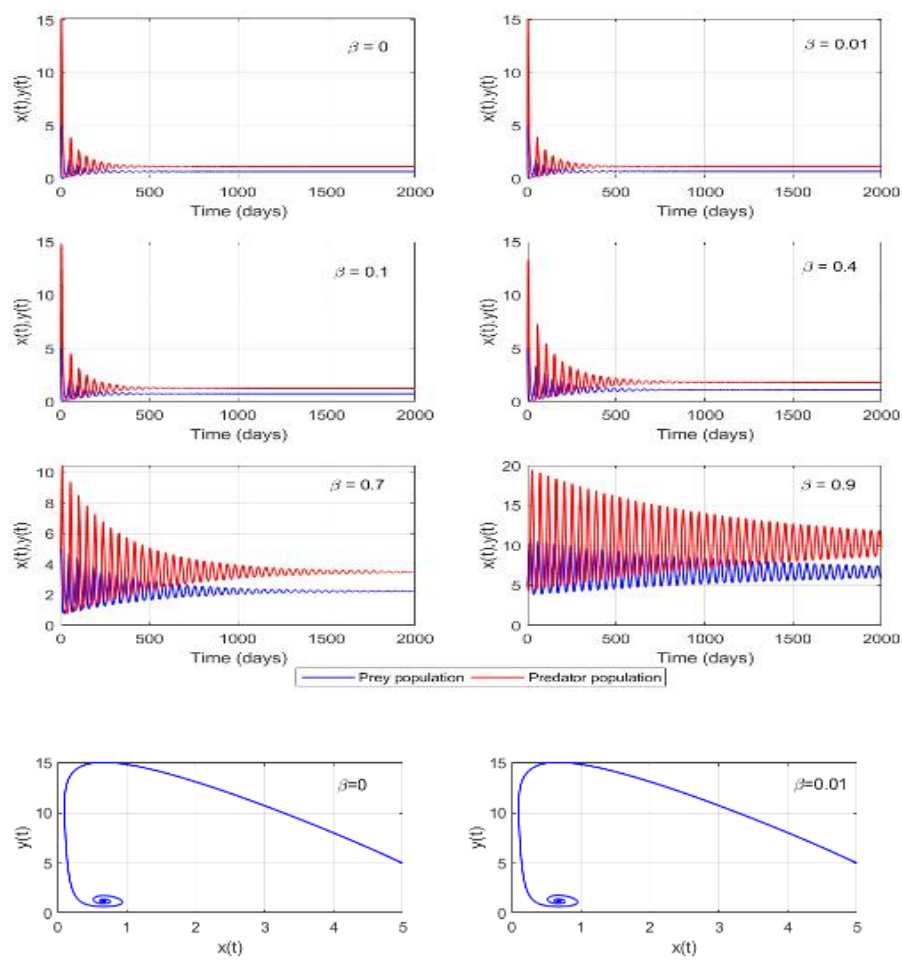

(b)
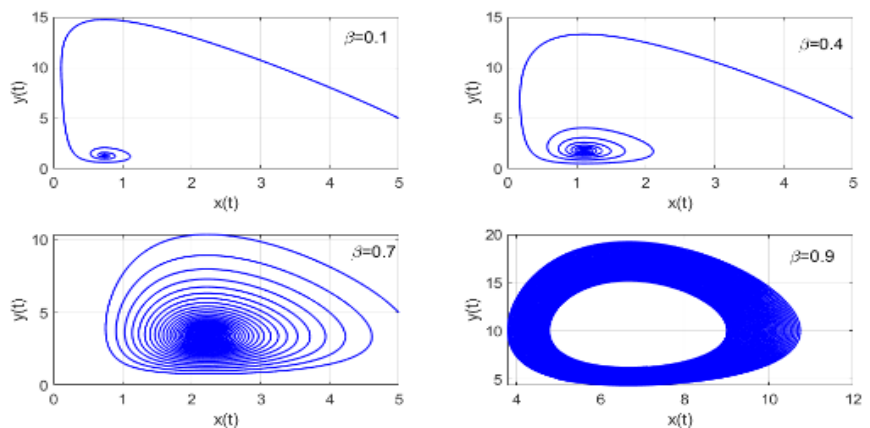

Figure 2: Long-term population dynamics (a) and phase plane plots (b) with Holling's type I functional response for $C(x)=c$ and $D(y)=0$. 
Stephano and Jung - Effects of refuge prey on stability of the prey-predator model ...

(a)
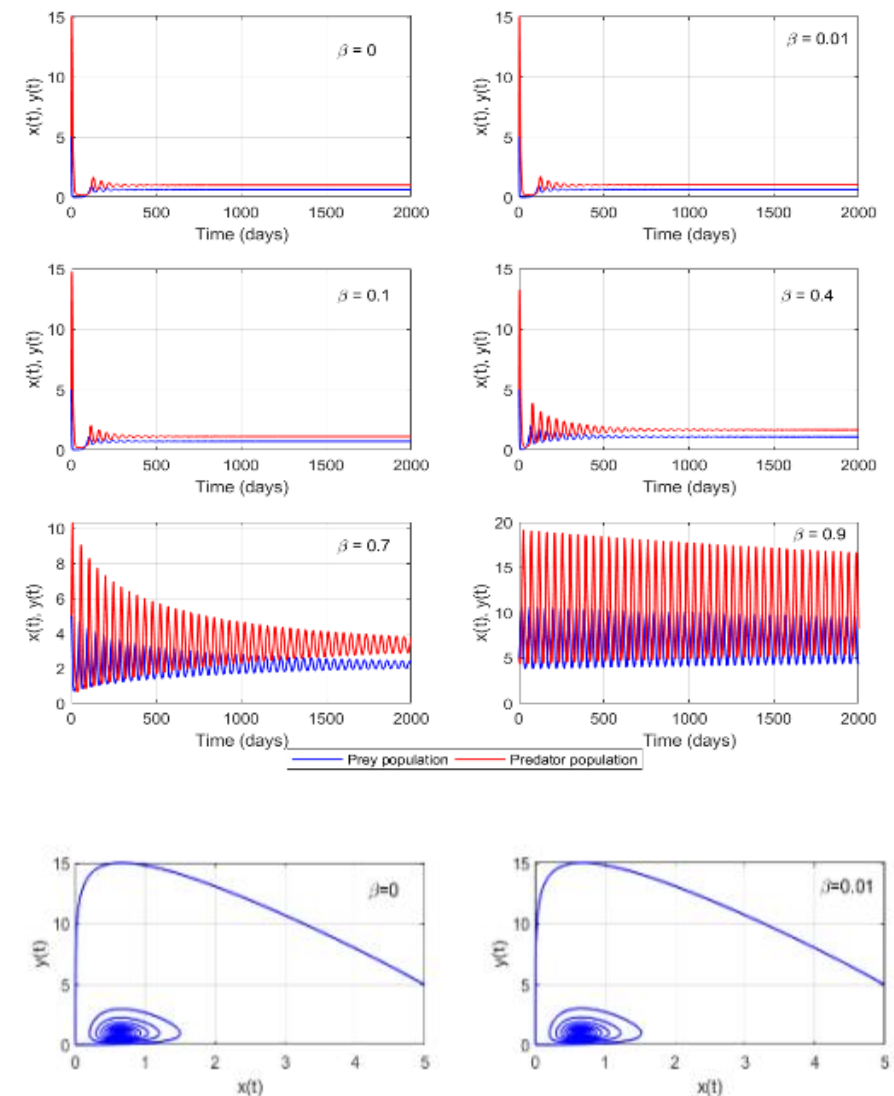

(b)
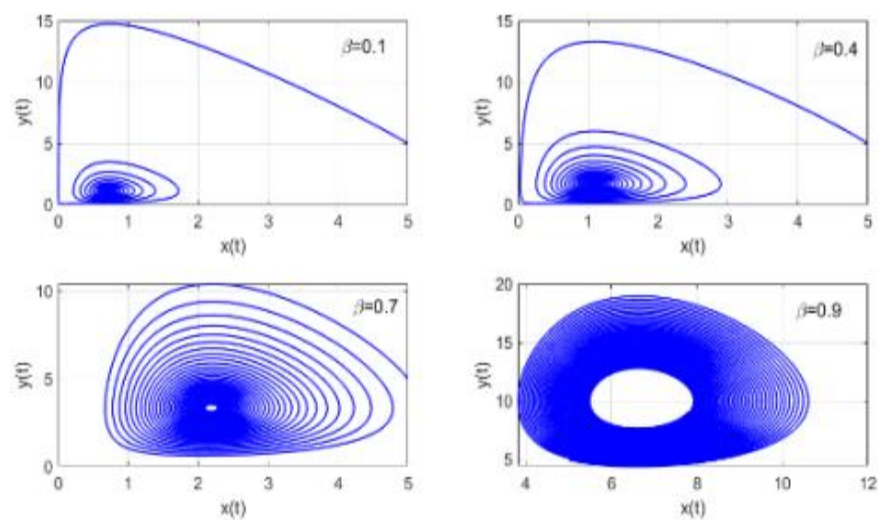

Figure 3: Long-term population dynamics (a) and phase plane plots with Holling's type I functional response for $C(x)=0$ and $D(y)=d$. 
(a)
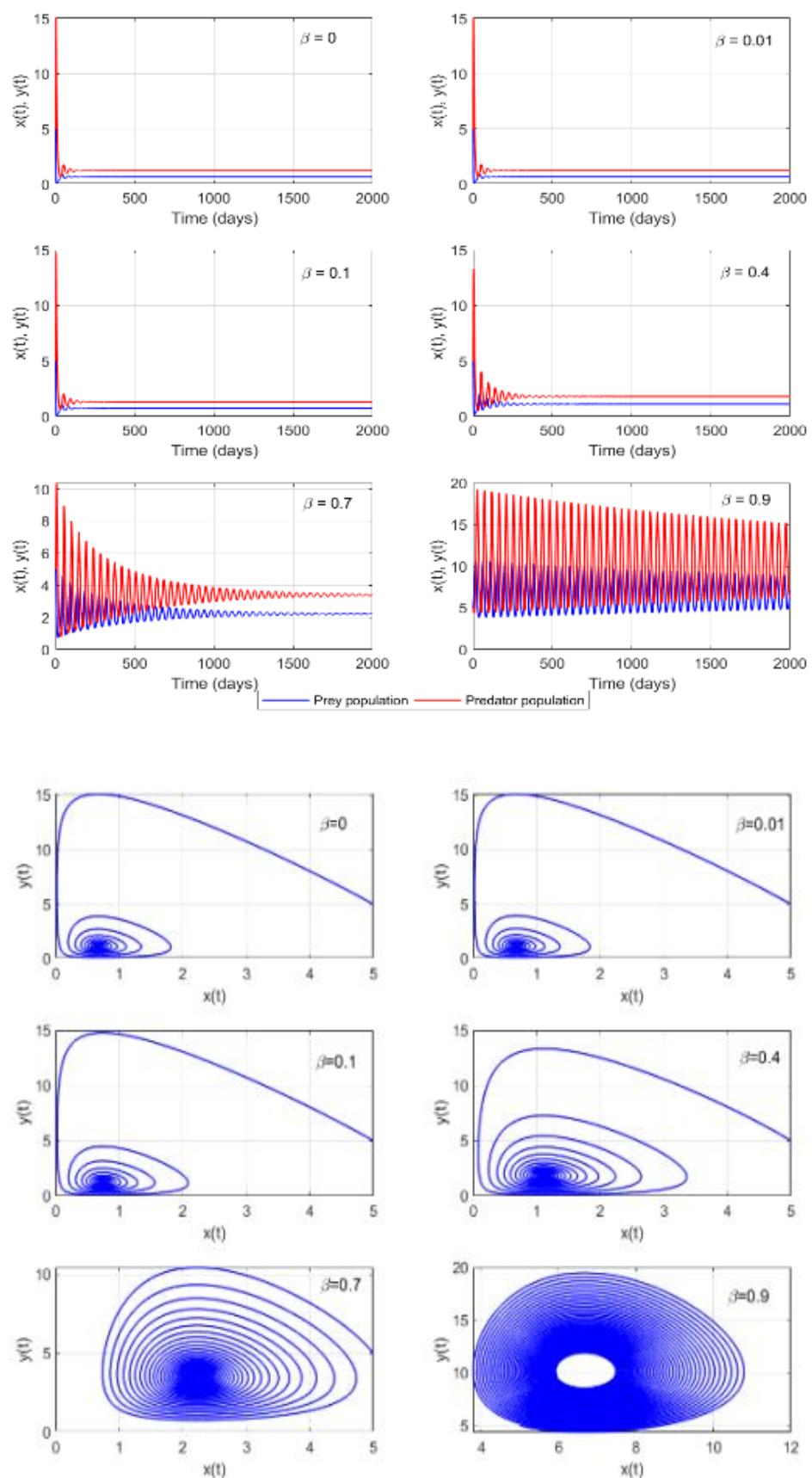

Figure 4: Long-term population dynamics (a) and phase plane plots (b) with Holling's type I functional response for $C(x)=\frac{c}{x}$ and $D(y)=0$. 
(a)
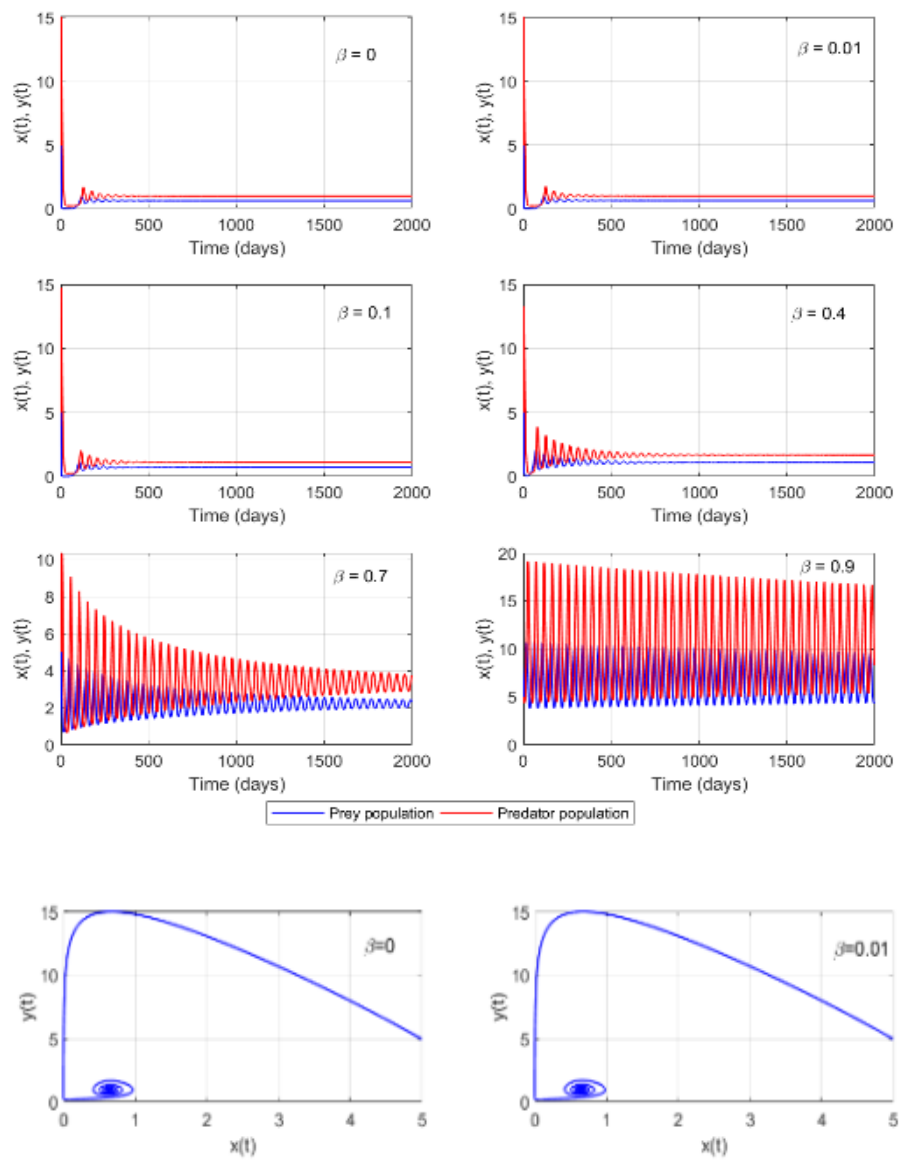

(b)
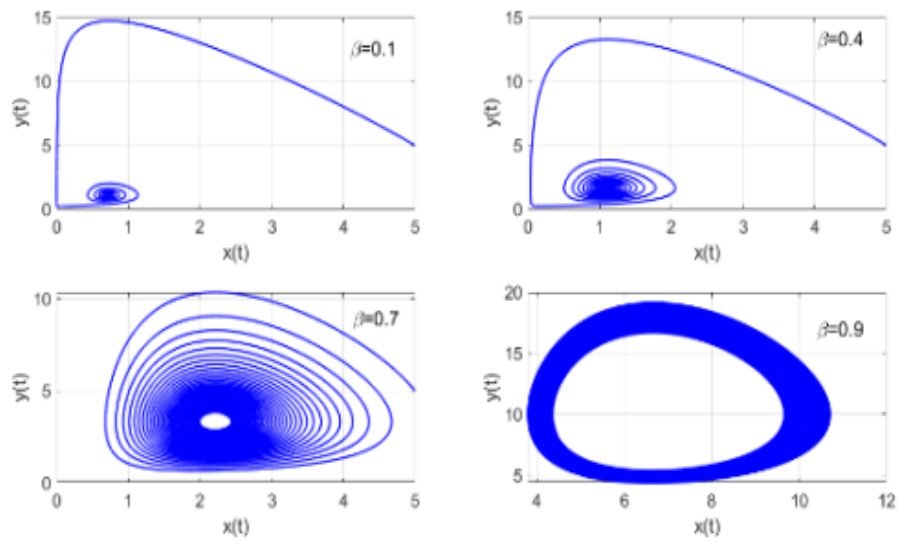

Figure 5: Long-term population dynamics (a) and phase plane plots (b) with Holling's type I functional response for $C(x)=0$ and $D(y)=\frac{d}{y}$. 
(a)
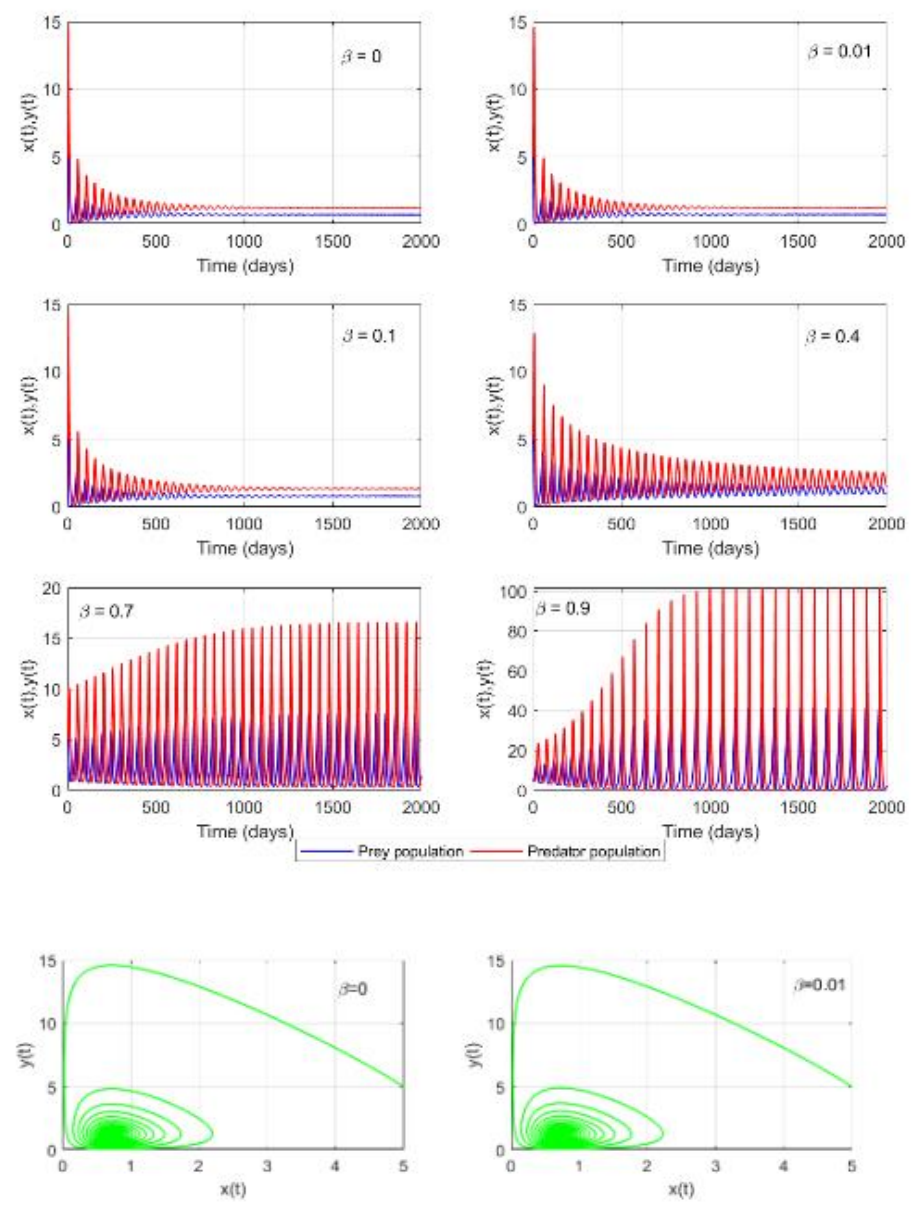

(b)
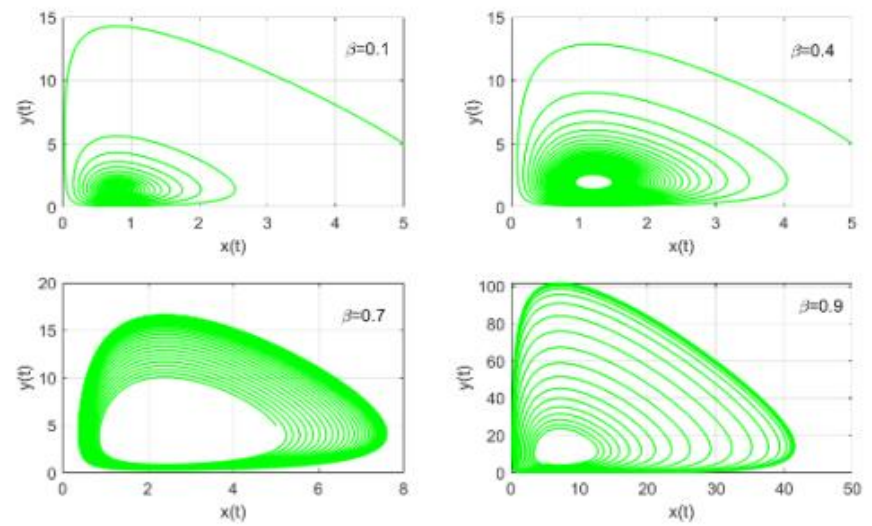

Figure 6: Long-term population dynamics (a) and phase plane plots (b) with Holling's type II functional response for $C(x)=c$ and $D(y)=0$. 
(a)
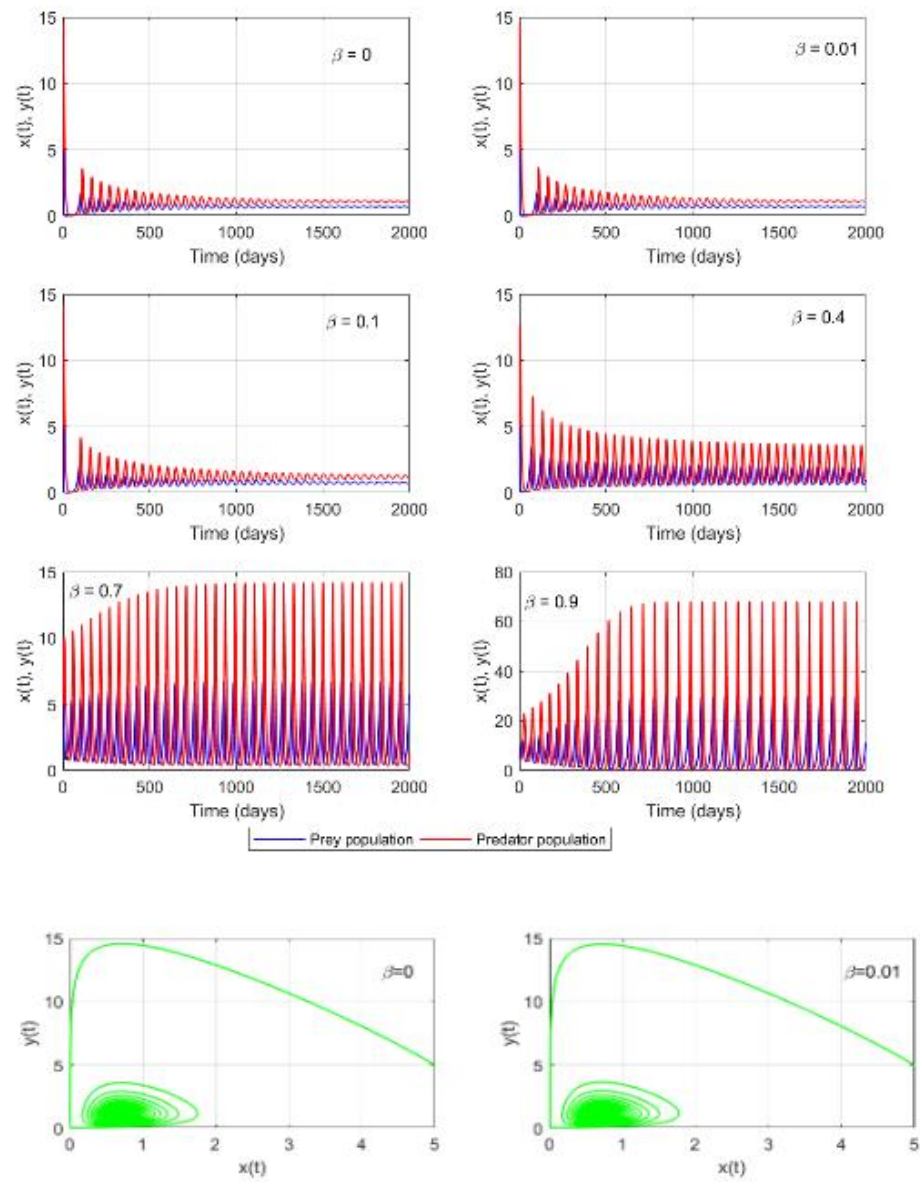

(b)
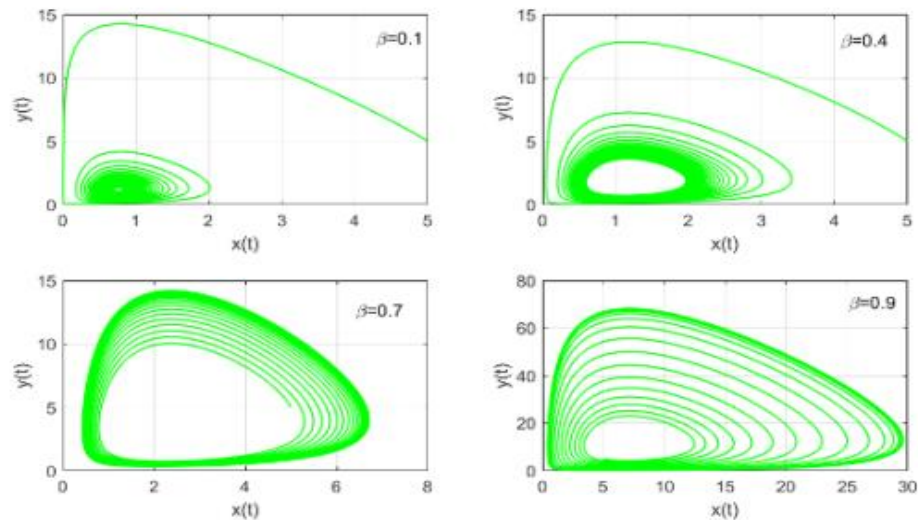

Figure 7: Long-term population dynamics (a) and phase plane plots (b) with Holling's type II functional response for $C(x)=0$ and $D(y)=d$. 

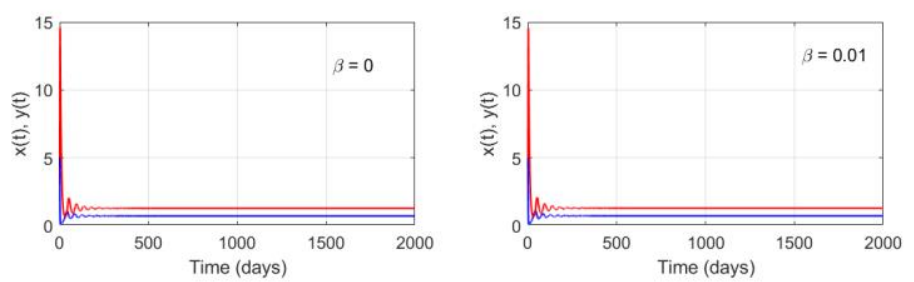

(a)
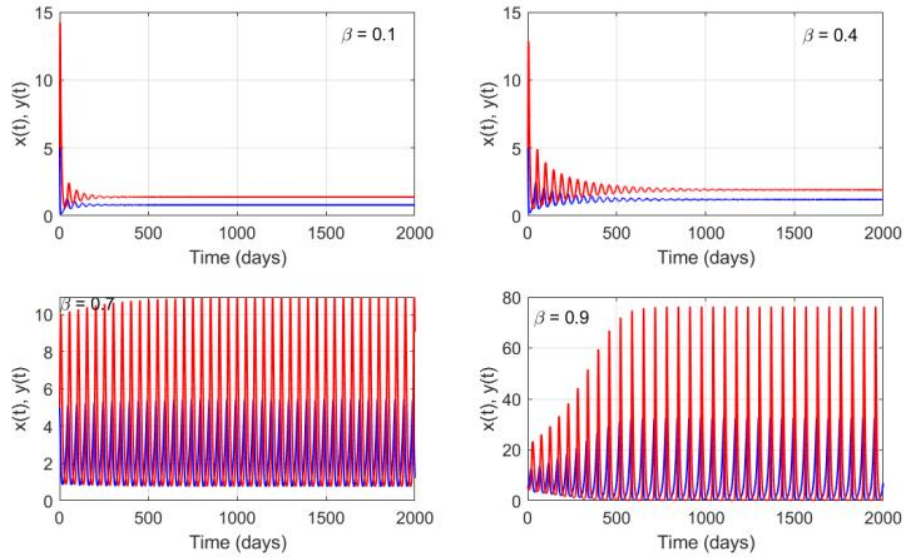

— Prey population ——Predator population

(b)
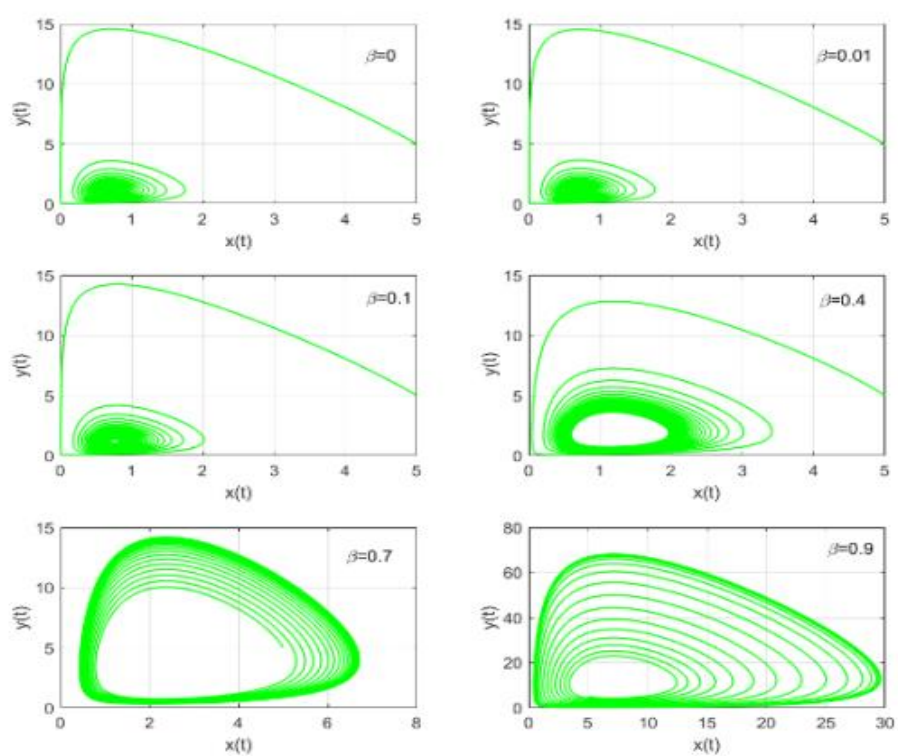

Figure 8: Long-term population dynamics (a) and phase plane plots (b) with Holling's type II functional response for $C(x)=\frac{c}{x}$ and $D(y)=0$. 

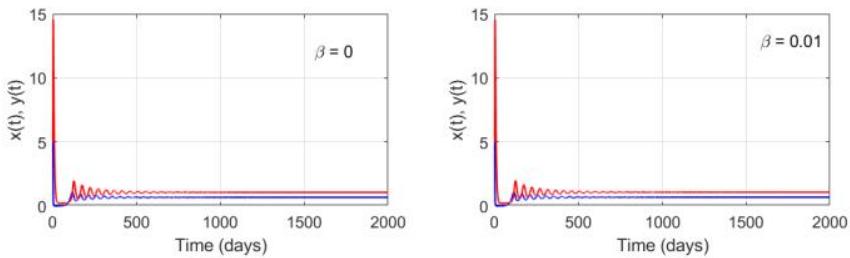

(a)
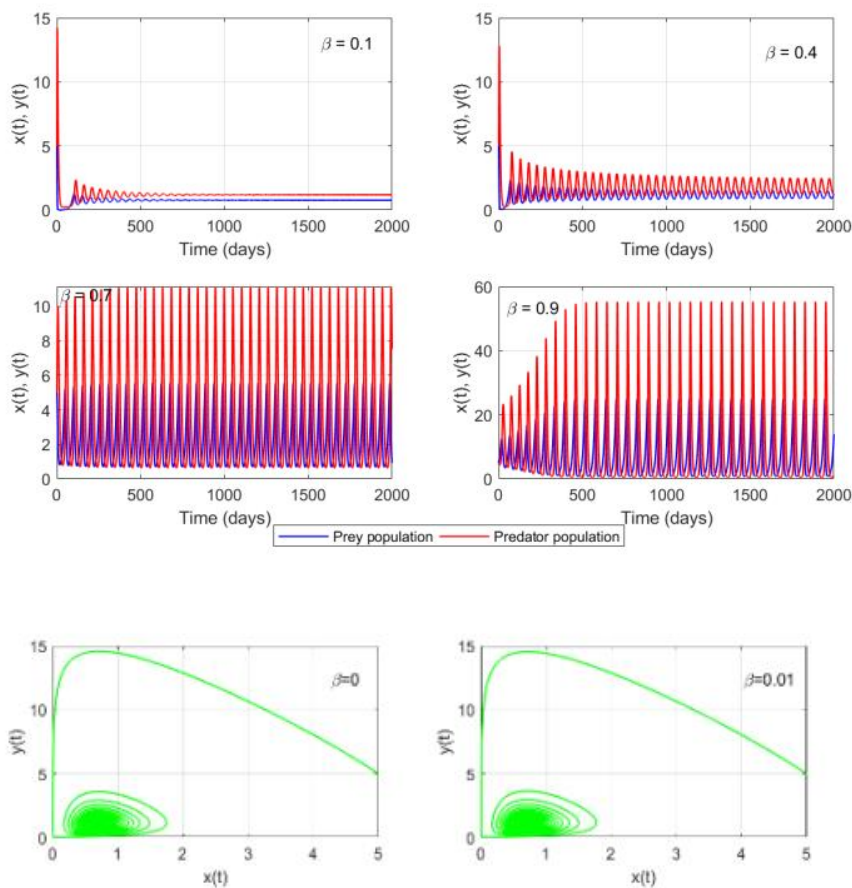

(b)
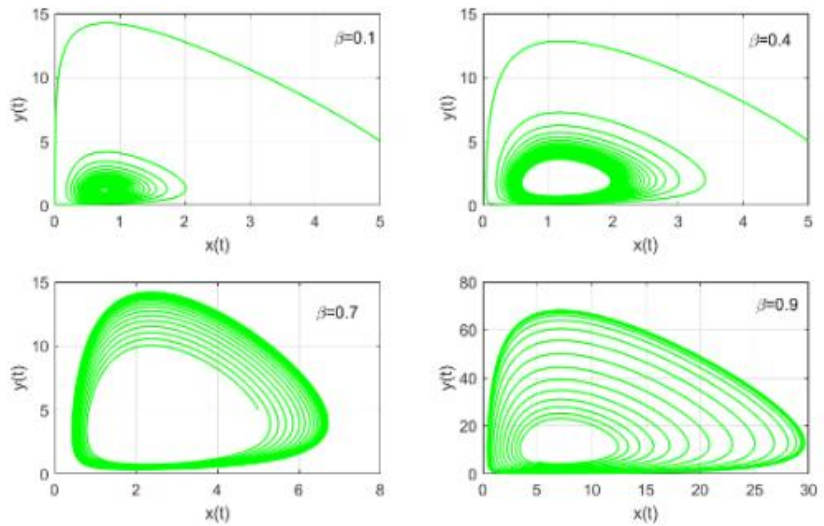

Figure 9: Long-term population dynamics (a) and phase plane plots (b) with Holling's type II functional response for $C(x)=0$ and $D(y)=\frac{d}{y}$. 

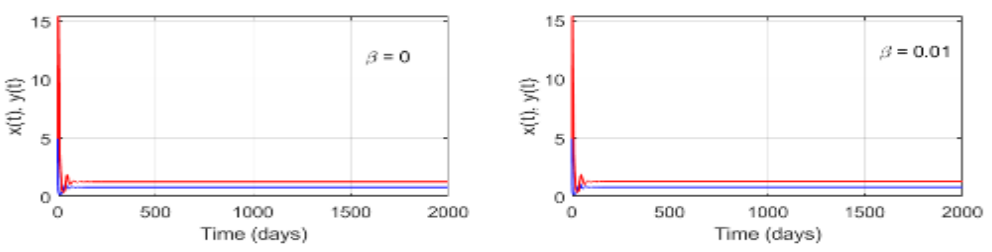

(a)
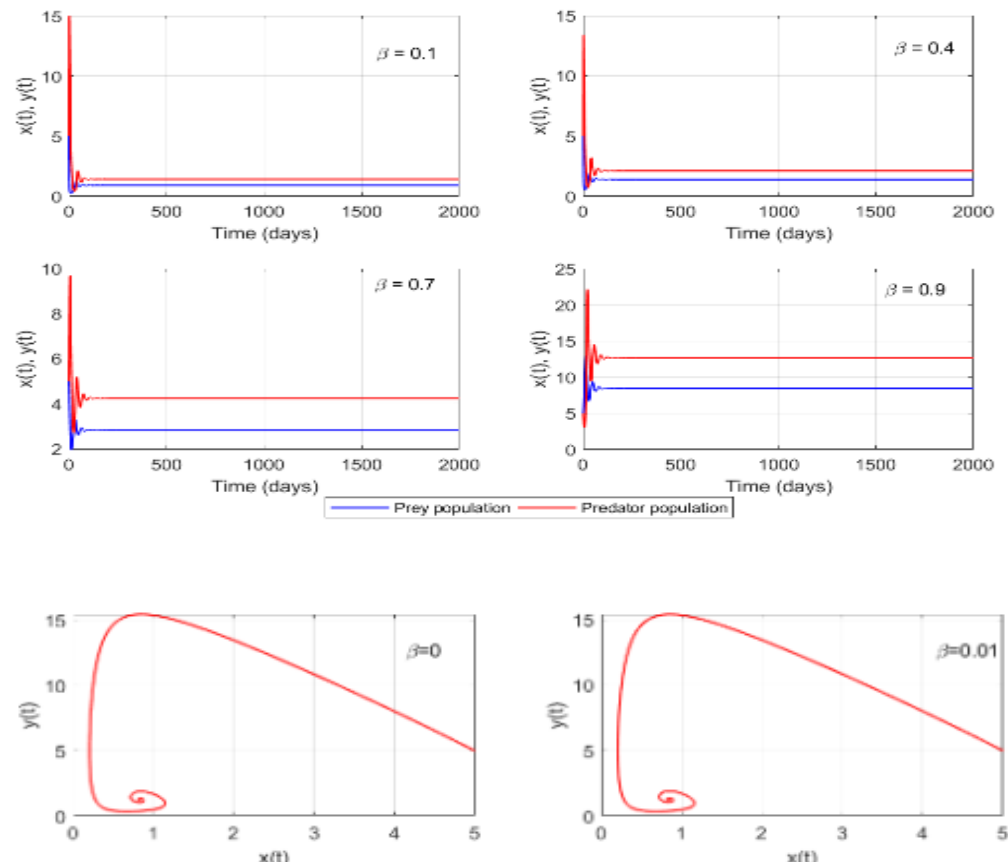

(b)
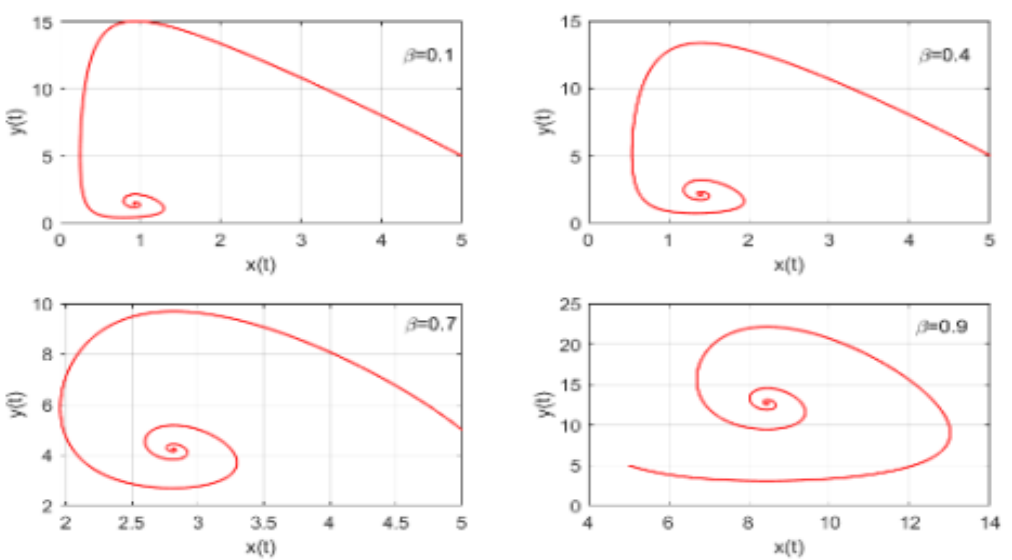

Figure 10: Long-term population dynamics (a) and phase plane plots (b) with Holling's type III functional response for $C(x)=0, c, \frac{c}{x}$ and $D(y)=0, d, \frac{d}{y}$. 
Stephano and Jung - Effects of refuge prey on stability of the prey-predator model ...

\section{Conclusion}

We presented a modified mathematical model that investigates the effects of refuge prey factor in a prey-predator system with immigrants. Through simulation, the prey factor indicated that the sensitivity of parameter $\beta$ determined by varying the values of its predefined range and immigrants factors $c$ and $d$ in all cases. The study found that a modified Lotkavoterra framework with Holling's type III functional response incorporated with refuge and immigration factors is asymptotically stable in all values of refuge factor within a range of $\beta$. However, the model with Holling's type I, the results indicated that is asymptotically stable when $\beta<r$ and unstable when with type II when $r<\beta<1$. We summarize the results as follows: (a) With Holling's type I functional response in a system, the proposed mathematical model is asymptotically convergent when a refuge prey factor is introduced. (b) The system with Holling's type II functional response, the proposed mathematical model is unstable and does not converge. (c) With Holling's type III functional response in a system, the proposed mathematical model is asymptotically stable.

We conclude with the following remarks: (i) Refuge prey factor has stabilizing and destabilizing effects. (ii) The likelihood of prey extinction declines if the proportion of prey in refuge population increases even if the predator population increases. Hence, the proposed mathematical model is valid, consistent and reliable for ecological studies. Therefore, a refuge factor is significant for ecological control of predator, however, increasing the amount of refuge can increase prey densities at a suitable limit. It balances the ecological dependence between prey and predator

\section{References}

Alinhac S 1995 Blowup for nonlinear hyperbolic equations, Progress. Nonlinear Differential Equations and Applications 17.

Allen LJ 2007 An introduction to mathematical biology. Upper Saddle River, New Jersey.

Berryman AA and Hawkins BA 2006 The refuge as an integrating concept in ecology and evolution. Oikos 115(1): 192-196.

Brown JH and Roughgarden J 1990 Ecology for a changing earth. Bull. Ecol.l Soc. Am. 173-188.

Cohen JE 1977 Ratio of prey to predators in community food webs. Nature 270(5633): 165-167.

Denny M 2014 Buzz Holling and the functional response. Bull. Ecol. Soc. Am. 95(3): 200-203.

Hansson LA and Åkesson S (Eds) 2014 Animal movement across scales. Oxford University press.

Holtmeier FK 2015 Animals' Influence on the Landscape and Ecological Importance. Netherlands: Springer.

Huang Y, Chen F and Zhong L 2006 Stability analysis of a prey-predator model with Holling type III response function incorporating a prey refuge. Appl. Math. Comput. 182(1): 672-683.

Kar TK 2005 Stability analysis of a preypredator model incorporating a prey refuge. Commun. Nonlinear Sci. Numer. Simul. 10(6): 681-691.

Kie JG 1999 Optimal foraging and risk of predation: effects on behavior and social structure in ungulates. J. Mammal. 80(4): 1114-1129.

May AC 1993 Protection of predator/prey diversity in the North Fork valley of the Flathead River: A case for land use planning.

Mukherjee D 2016 The effect of refuge and immigration in a predator-prey system in the presence of a competitor for the prey. Nonlinear Anal.: Real World Appl. 31: 277-287. 
Nachman G 1988 Regional persistence of locally unstable predator/prey populations. Exp. Appl. Acarol. 5(3-4): 293-318.

Parmesan C 2006 Ecological and evolutionary responses to recent climate change. Annu. Rev. Ecol. Evol. Syst. 37: 637-669.

Petchey OL 2000 Prey diversity, prey composition, and predator population dynamics in experimental microcosms. $J$. Anim. Ecol. 69(5): 874-882.

Robinson NM, Leonard SW, Ritchie EG, Bassett M, Chia EK, Buckingham S, Gibb H, Bennett AF and Clarke MF 2013 Refuges for fauna in fire-prone landscapes: their ecological function and importance. $J$. Appl. Ecol. 50(6): 1321-1329.

Sih A 1987 Prey refuges and predator-prey stability. Theor. Popul. Biol. 31(1): 1-12.
Singh UP and Singh AK 1999 Human Ecology \& Development in India, APH Publishing.

Takeru T, Gavina MKA, Kawano T, Tubay JM, Rabajante JF, Ito H, Morita S, Ichinose G, Okabe T, Togashi T and Tainaka KI 2018 Asymptotic stability of a modified LotkaVolterra model with small immigrations. Sci. Rep. 8(1): 1-7.

Thomas R 2019 Marine Biology: An Ecological Approach. Scientific eResources.

Wertheim B, van Baalen EJA, Dicke M and Vet LE 2005 Pheromone-mediated aggregation in nonsocial arthropods: evolutionary ecological perspective. Аnпи. Rev. Entomol. 50: 321-346. 